\title{
A study of local time and longitudinal variability of the amplitude of the equatorial electrojet observed in POGO satellite data
}

\author{
H. R. Kim* and Scott D. King \\ Department of Earth and Atmospheric Sciences, Purdue University, West Lafayette, IN 47907, U.S.A.
}

(Received May 28, 1998; Revised April 19, 1999; Accepted April 19, 1999)

\begin{abstract}
The local time and longitudinal variations of the amplitude of the equatorial electrojet (EE) are investigated using the POGO series satellite data. An iterative normalization scheme is developed to separate the variations of the amplitude of the EE with respect to longitude and local time. The resulting local time variation of the EE compares favorably with observatory based studies; however, the longitudinal variation is not well resolved. The altitude of the satellite is another factor that affects the amplitude of the EE. We use a simple linear scaling of amplitude of the EE with the inverse of the satellite altitude. An experiment including only the satellite passes below $450 \mathrm{~km}$ altitude indicates that there may be problems in correcting the data from higher altitudes. The reason behind the discrepancy between the results from the data limited to below $450 \mathrm{~km}$ and the full data set is not understood and requires further investigation.
\end{abstract}

\section{Introduction}

The equatorial electrojet (EE), an anomalous variation of the geomagnetic field, results from an enhanced east-west current in a narrow belt centered on the magnetic dip equator. The current system that produces the EE feature is approximately $110 \mathrm{~km}$ above the surface of the Earth. The basic physics of a horizontally-stratified ionosphere near the magnetic dip equator, where the magnetic field is nearly horizontal, is reasonably well understood; however, the observations of the $\mathrm{EE}$ are limited to a handful of near equator observatories and several satellite missions. Forbes (1981) and Rastogi (1989) reviewed the basic theory and the observatory data pertinent to the EE, while Onwumechili (1985) reviewed the satellite observations.

Cain and Sweeney (1973) described the variations of the EE using the POGO series satellite data. The equatorial passes in their study range from 400 to $800 \mathrm{~km}$ in altitude and include local times between 0900 to 1500 . They found that, as expected, the minimum of the $\mathrm{V}$-shaped equatorial electrojet signature was within 0.5 degrees of the position of the dip equator, and that the magnitude of the electrojet signature varied with the longitude, with a peak in amplitude at approximately $60^{\circ} \mathrm{W}$ and a secondary peak at approximately $100^{\circ}$ E. Agu and Onwumechili (1981) found a variation in the current intensity, half-width of the V-shaped feature, and total eastward current of the EE with the local time. Onwumechili and Agu (1980) found a variation of the EE with the satellite altitude. Doumouya et al. (1998) report variations in the magnitude of the EE with respect to the local time, based

*Now at Department of Geological Sciences, Ohio State University, Columbus, $\mathrm{OH} 42325$, U.S.A.

Copy right $(\mathrm{C})$ The Society of Geomagnetism and Earth, Planetary and Space Sciences (SGEPSS); The Seismological Society of Japan; The Volcanological Society of Japan; The Geodetic Society of Japan; The Japanese Society for Planetary Sciences. on the data from ground based magnetotelluric stations in Africa. Maeda et al. (1982) and Roy (1983) observed the EE in the Magsat data, which is limited to dusk and dawn local times.

Based on the previous investigations of the EE, the present paper will focus on two aspects of the satellite observations of the EE. The first is a discussion of the local time variation of the EE from the POGO data, including a comparison of our results based on the satellite observations with the model based on the magnetic observatory data (Gupta and Chapman, 1970; Forbes and Lindzen, 1976). The second aspect of this paper is an attempt to separate the longitudinal variation of the amplitude of the EE from the local time variation of the amplitude of the EE using an iterative normalization technique.

\section{Data Processing}

We use the POGO satellite series (OGO 2, 4, and 6) measurements because the POGO satellites acquired data at all local times; thus the data set contains tracks from almost all longitudes and local times. The POGO satellites (specifically OGO-4 and OGO-6 which are used in this study) collected data between 1967-1970. Using the POGO data Langel (1990) has estimated that the standard deviation of error estimates from all the known sources of error for the POGO data set is $5.63 \mathrm{nT}$. In order to ensure that our field observations are not contaminated by magnetic disturbances, we consider only those satellite passes with the $K p$ index less than $1+$. After applying the $K p$ less than $1+$ criterion for data quality, and eliminating satellite passes where the average altitude of the satellite is greater than $800 \mathrm{~km}$, there are 225 satellite passes remaining in our data set. Out of those 225 satellite passes, 57 passes contain a recognizable EE feature at the dip equator. The month, altitude, longitude, local time and EE amplitude of the 57 satellite tracks are provided in Table 1. 
Table 1. Parameters for the 57 tracks used in this study.

\begin{tabular}{|c|c|c|c|c|}
\hline Month & Altitude (km) & Local time & Longitude & EE amplitude* (nT) \\
\hline 9 & 415.12 & 16.93 & -179.39 & 4.34 \\
\hline 7 & 430.03 & 12.88 & -172.43 & 10.05 \\
\hline 9 & 419.98 & 16.80 & -172.04 & 4.14 \\
\hline 9 & 412.61 & 16.17 & -168.34 & 5.36 \\
\hline 7 & 427.73 & 13.71 & -166.97 & 13.29 \\
\hline 5 & 423.29 & 9.78 & -160.07 & 5.76 \\
\hline 1 & 720.37 & 13.01 & -159.96 & 10.50 \\
\hline 7 & 416.06 & 15.25 & -156.04 & 6.17 \\
\hline 9 & 413.16 & 16.29 & -152.66 & 5.36 \\
\hline 5 & 430.88 & 10.97 & -152.61 & 13.26 \\
\hline 4 & 761.34 & 14.82 & -146.87 & 5.27 \\
\hline 9 & 416.18 & 17.16 & -146.66 & 3.65 \\
\hline 9 & 412.73 & 16.53 & -142.98 & 3.17 \\
\hline 7 & 421.39 & 14.04 & -142.34 & 11.38 \\
\hline 4 & 743.14 & 14.11 & -136.21 & 3.57 \\
\hline 1 & 732.59 & 12.76 & -135.29 & 13.59 \\
\hline 7 & 421.92 & 15.58 & -131.43 & 8.01 \\
\hline 9 & 413.10 & 16.64 & -127.35 & 2.52 \\
\hline 4 & 410.48 & 13.28 & -127.26 & 5.66 \\
\hline 9 & 415.01 & 16.62 & -121.33 & 3.99 \\
\hline 9 & 414.07 & 16.89 & -117.67 & 3.07 \\
\hline 4 & 415.68 & 13.66 & -102.04 & 5.37 \\
\hline 10 & 787.81 & 9.26 & -77.35 & 15.86 \\
\hline 5 & 413.39 & 11.31 & -57.97 & 11.51 \\
\hline 3 & 412.04 & 8.29 & -55.21 & 5.36 \\
\hline 10 & 787.50 & 9.63 & -52.82 & 20.45 \\
\hline 2 & 443.59 & 11.54 & -44.39 & 11.40 \\
\hline 7 & 649.06 & 14.71 & -36.46 & 7.34 \\
\hline 8 & 779.46 & 10.11 & -34.26 & 10.40 \\
\hline 9 & 431.11 & 13.44 & -33.79 & 5.47 \\
\hline 2 & 443.21 & 11.30 & -27.36 & 18.31 \\
\hline 6 & 453.34 & 16.31 & -24.38 & 1.90 \\
\hline 2 & 444.62 & 10.91 & -19.95 & 17.10 \\
\hline 2 & 475.73 & 8.87 & -19.93 & 4.80 \\
\hline 5 & 409.13 & 9.76 & -18.79 & 4.57 \\
\hline 10 & 633.30 & 11.68 & -11.98 & 8.05 \\
\hline 4 & 514.81 & 15.75 & -6.60 & 5.61 \\
\hline 2 & 444.94 & 10.64 & -3.05 & 29.00 \\
\hline 2 & 449.10 & 11.24 & 4.36 & 16.06 \\
\hline 7 & 426.04 & 14.30 & 10.24 & 2.65 \\
\hline
\end{tabular}


Table 1. (continued)

\begin{tabular}{ccccc}
\hline Month & Altitude $(\mathrm{km})$ & Local time & Longitude & EE amplitude* $(\mathrm{nT})$ \\
\hline 4 & 529.88 & 15.49 & 11.00 & 1.22 \\
9 & 426.07 & 14.24 & 15.47 & 7.11 \\
9 & 424.60 & 13.97 & 64.56 & 3.44 \\
8 & 503.12 & 10.62 & 65.24 & 8.94 \\
7 & 589.03 & 15.21 & 80.75 & 7.58 \\
9 & 425.34 & 13.35 & 89.13 & 3.00 \\
7 & 460.57 & 14.23 & 91.96 & 7.41 \\
7 & 443.64 & 14.35 & 94.11 & 6.32 \\
5 & 421.16 & 10.44 & 100.62 & 13.37 \\
7 & 469.55 & 13.34 & 103.35 & 7.68 \\
7 & 590.41 & 15.35 & 105.80 & 7.30 \\
4 & 527.43 & 16.00 & 108.83 & 2.49 \\
5 & 421.99 & 10.79 & 125.52 & 15.59 \\
7 & 418.54 & 13.23 & 137.98 & 5.63 \\
5 & 436.38 & 9.06 & 154.70 & 1.98 \\
7 & 421.44 & 12.56 & 162.91 & 9.20 \\
7 & 411.48 & 15.23 & 179.60 & 5.45 \\
\hline
\end{tabular}

*data set: Direct-CC.

Passes from the nighttime and predawn local times do not contain an EE feature; however, there are also passes in the daytime where the EE feature is not observed. The absence of the EE signature in the daytime passes was noted by Cain and Sweeney (1973) but is not clearly understood.

The amplitude of the EE has also been shown to vary with the season (e.g., Cain and Sweeney, 1973; Langel et al., 1993; Ravat and Hinze, 1993). Due to the limited number of satellite passes that remain after the $K p<1+$ selection criteria is applied to the POGO data set, and the distribution of these satellite passes across all seasons, we do not attempt to account for seasonal effects. We would not have enough observations for a meaningful analysis. We plotted our final results by season to investigate the possibility that seasonal variations were being masked into the local time or longitudinal variations that we model due to a clustering of the data by seasons.

Working with the 225 satellite passes that meet the low noise and altitude criterion, we remove the main field using the model POGO (2/72), which includes spherical harmonics up to degree and order 13 for both the constant and the first time-derivative terms (Langel et al., 1980). We use a highpass Kaiser filter with a cutoff wavelength of $12,000 \mathrm{~km}$ on a pass-by-pass basis following Langel et al. (1993). The filtering allows us to isolate the fields associated with the EE or other ionospheric fields (Maeda et al., 1982), which are intermediate wavelength features, while removing the effects of the longer wavelength magnetospheric ring current and the solar quiet variations.
Separating the crustal field and the ionospheric field presents a challenge. While filtering allows us to eliminate the long wavelength fields associated with the magnetospheric ring current and solar quiet variations, the amplitudes and wavelengths of the ionospheric and crustal fields are similar. In order to isolate the ionospheric contribution to the magnetic field, we subtract a crustal field model derived by the equivalent source point technique (Langel, 1990) using the averaged POGO data; however, it is possible that removing a crustal field model may eliminate, or at least underestimate, the amplitude of the EE (Langel, 1993). Because the crustal field model may contain some of the EE signal we are looking for, we create two data sets: in the first we subtract the main field model from the POGO data and then apply the long-wavelength filtering correction described above. For convenience we will refer to this data set as 'NOCC.' For the second data set we subtract the main field model from the POGO data, apply the long wavelength filtering correction, and subtract the crustal field model. We will refer to this data set as 'CC.' By comparing these two data sets, we can investigate whether the crustal correction affects our models of the amplitude variation of the EE.

\section{Measurements of the EE and Data Analysis}

As a satellite pass crosses the dip equator in daylight, the value of the residual field drops to a local minimum. This inverted V-shaped feature, marked by CAD in panel (a) of Fig. 1, is the characteristic signature of the equatorial electrojet (Onwumechili and Agu, 1980). 
(a)

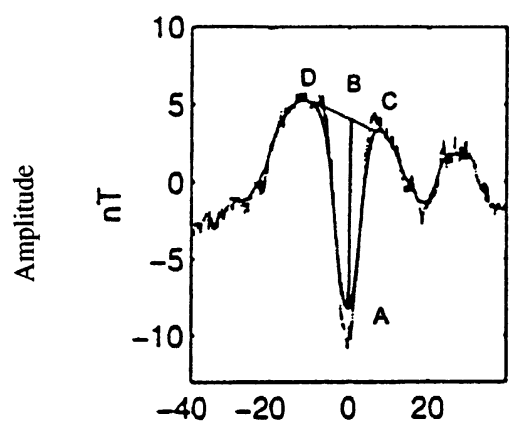

(b)

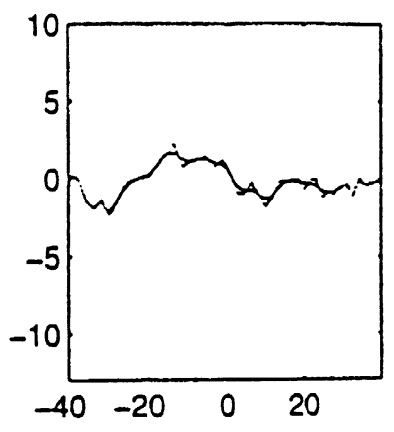

Dip latitude

(c)

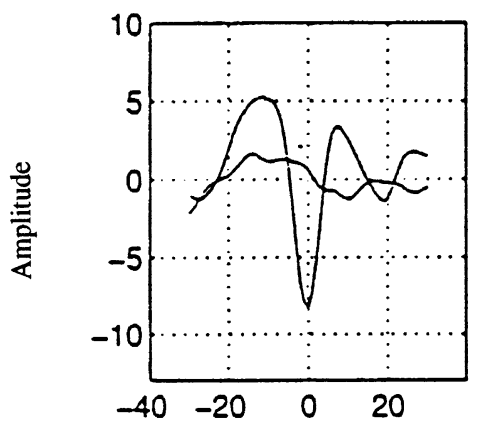

(d)

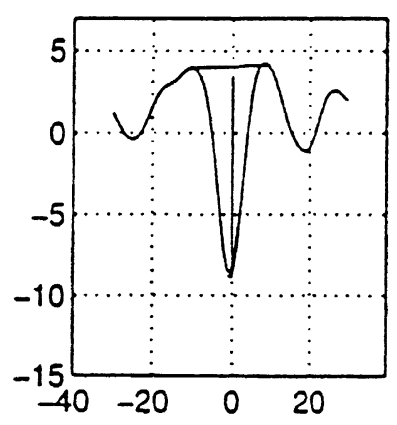

Dip latitude

Fig. 1. A procedure to measure the EE strength from the satellite pass by subtracting a pass near midnight from a pass containing the EE. Panel (a) shows the pass of the EE at $1250 \mathrm{LT}$ above longitude $172.4^{\circ} \mathrm{W}$ at the altitude of $430 \mathrm{~km}$. CAD is the EE signature. The minimum point A occurs over the dip equator. Panel (b) shows the neighboring pass with no EE feature at $0118 \mathrm{LT}$ above longitude $173.7^{\circ} \mathrm{W}$ at the altitude of $411 \mathrm{~km}$. Panel (c) presents these two passes together. Panel (d) shows the difference between these two passes.

Following Onwumechili and Agu, the magnitude of the signature is estimated by measuring the length of the line $\mathrm{AB}$ in nanotesla (nT). Because we remain skeptical about the source of the signature we measure at this point, we devised a second measuring technique. In our new technique, a second satellite pass with a longitude, latitude, and altitude as close as possible to the original satellite pass is selected (panel (b) in Fig. 1). The local time of the second satellite pass is near midnight, thus no ionospheric field should be present. We assume that any signal that we observe in the nighttime pass represents an unmodeled crustal field component that is also present in the daytime pass. The nighttime pass is subtracted from the daytime pass (panel (c) in Fig. 1) and the resulting modified pass (panel (d) in Fig. 1) is measured using the original measurement technique.

Both of these measurement techniques are applied to each of the data sets described in the previous section (i.e., NOCC and $\mathrm{CC}$ ). This provides an additional means of assessing the effect of the crustal field model on the amplitude of the EE. For convenience, we refer to the first method as the "Direct Measurement," and the second method as the "Pass Difference Measurement." The two measurements techniques are applied to the two data sets described in the previous section, producing four data sets.

As a first step toward investigating the influence of the crustal model on the data, we compute the correlation coeffi-
Table 2. The correlation coefficients for the data sets.

\begin{tabular}{cccc}
\hline Direct-CC & Direct-NOCC & PD-CC & PD-NOCC \\
\hline 1.000 & 0.977 & 0.971 & 0.970 \\
0.977 & 1.000 & 0.965 & 0.957 \\
0.971 & 0.965 & 1.000 & 0.992 \\
0.970 & 0.957 & 0.992 & 1.000 \\
\hline
\end{tabular}

cients for the EE amplitudes from each data set (correlating a single data set with each of the other data sets separately). The correlation coefficients are presented in Table 2. The first thing to notice is that the choice of the measurement technique (Direct or Path Difference) and the inclusion or exclusion of the crustal field correction (CC or NOCC) have only a minor effects on the values of the correlation coefficients. On further inspection, we note that the correlation coefficients are smallest when correlating the data set using the Direct Measurement technique without the crustal field correction (Direct-NOCC) with any of the other data sets. The data set Direct-NOCC is the only data set where there has been no attempt to remove a crustal field contribution from the data. The fact that the smallest correlation coefficients occur when one of the other three data sets is correlated 
with Direct-NOCC suggests that there is a measurable crustal field component in our data; however, because the correlation coefficients are still quite large, it suggests that this crustal field component has only a minor impact on the amplitude of the EE.

The observation of the EE from each satellite pass is from a different altitude. In our data set the altitude of the satellite ranges from 408 to $792 \mathrm{~km}$. In an attempt to correct the amplitude of the EE for the effect of the satellite altitude, we normalize the satellite passes to a reference altitude by multiplying the amplitude of the magnetic field from the satellite pass by the factor $\mathrm{ALT}_{i} / \mathrm{ALT}^{\prime}$, where $\mathrm{ALT}_{i}$ is the altitude of the $i$ th satellite pass and $\mathrm{ALT}^{\prime}$ is the average altitude for the whole data set (Kane, 1973). For our data set, $\mathrm{ALT}^{\prime}$ is $550 \mathrm{~km}$.

\section{Iterative Normalization Technique}

After the altitude normalization, the four data sets are used to investigate the variation of the amplitude of the EE with respect to the local time and longitude. The previous studies of the longitudinal variation of the amplitude of the EE from the POGO data (Cain and Sweeney, 1973) did not consider the local time variation of the EE. Yet even a cursory look at our data sets shows that there is a strong dependence of the EE amplitude on the local time. Unfortunately, there are not enough observations to take a small time window and study the longitudinal variations from a nearly uniform local time.

The problem we are faced with is the need to simultaneously fit the data to both the local time and longitudinal variations of the data without knowing the functional form of either variation. We attempt to solve this problem by normalizing the data to a fixed value for one of the variations (i.e., a fixed time or a fixed longitude) and solving for the other variation. The outline of this procedure has the following steps:

1) We estimate both the longitude and local time variations independently from the original data sets (working with one data set at a time). We choose to fit the longitudinal variation of the data to a four-term harmonic expansion of the form:

$$
S(\phi)=\sum_{n=0}^{3} A_{n} \cos \left(n \phi+\Theta_{n}\right)
$$

where $S(\phi)$ is the approximation of the amplitude with the longitude, $\phi$, and $A_{n}$ and $\Theta_{n}$ are the amplitude and phase of the $n$th harmonic term. Similarly for the local time variation of the data, we use a five-term expansion of the form:

$$
T(t)=\sum_{n=0}^{4} B_{n} \cos \left(n t+\Delta_{n}\right)
$$

where $T(t)$ is the approximation of the amplitude with the local time, $t$, and $B_{n}$ and $\Delta_{n}$ are the amplitude and phase of the $n$th harmonic term.

2) To account for the longitudinal variation of the data in our local time fit, we normalize the data to a single longitude using our initial fit to the longitude function from step (1), i.e.,

$$
\operatorname{data}_{\phi_{o}}(t)=\operatorname{data}(\phi, t) \times\left(\frac{S\left(\phi_{o}\right)}{S(\phi)}\right)
$$

where $\operatorname{data}(\phi, t)$ is the original data, $\operatorname{data}_{\phi_{o}}(t)$ is the data set with the first approximation of the longitudinal variation removed, and $\phi_{o}$ is the arbitrary longitude to which the data set is normalized. We then fit a function with the same the form as Eq. (1) to the 'normalized' data set, $\operatorname{data}_{\phi_{o}}(t)$. We call this new function $T_{1}(\phi)$.

3) To account for the local time variation of the data in our longitudinal fit, we normalize the data to a single local time using our initial fit to the local time function from step (1), i.e.,

$$
\operatorname{data}_{t_{o}}(\phi)=\operatorname{data}(\phi, t) \times\left(\frac{T\left(t_{o}\right)}{T(t)}\right)
$$

where $\operatorname{data}(\phi, t)$ is the original data, $\operatorname{data}_{t_{o}}(\phi)$ is the data set with the first approximation of the local time variation removed, and $t_{o}$ is the arbitrary longitude to which the data set is normalized. We then fit a function with the same form as Eq. (2) to the 'normalized' data set, $\operatorname{data}_{t_{o}}(\phi)$. We call this new function $S_{1}(\phi)$.

4) We repeat the procedure outlined in steps (2) and (3), now using the local time and longitude functions $S_{1}(\phi)$ and $T_{1}(t)$ in place of $S(\phi)$ and $T(t)$ in Eqs. (3) and (4). We repeat this procedure until the function from one iteration to the next does not change, or until the function oscillates between two solutions (which is what happens in most of the cases). It is important to recognize that when we normalize the data in steps (2) and (3), we are always working with the original data. In other words, data $(\phi, t)$ on the right hand side of Eqs. (3) and (4) is always the original data set, never a 'normalized' data set. Only the functional form of the local time or longitudinal variation changes.

This procedure is applied independently to each of the four data sets described in the previous section. Figure 2 illustrates the results of the normalization procedure for the local time variation. The coefficients of Eqs. (1) and (2) after the second iteration are provided in Table 3.

Figure 2 panel (a) shows the data from the direct measurement technique with the crustal correction (Direct-CC). The original data are plotted as circles and the original local

Table 3. Coefficients for Eqs. (1) and (2) for model in Figs. 2 and 3 after the second iteration $\left(\Theta_{n}\right.$ and $\Delta_{n}$ are in Radians).

\begin{tabular}{rrrrr}
\hline$n$ & $A_{n}(\mathrm{nT})$ & \multicolumn{1}{c}{$\Theta_{n}$} & $B_{n}(\mathrm{nT})$ & \multicolumn{1}{c}{$\Delta_{n}$} \\
\hline 0 & 2.3877 & 0.0 & 9.8444 & 0.0 \\
1 & -4.0784 & -6.1764 & -3.7408 & -4.7510 \\
2 & 2.4681 & 0.3750 & -2.4765 & 5.5927 \\
3 & -1.3281 & -5.0520 & 1.7310 & -0.0065 \\
4 & -1.4162 & 5.2529 & 0.0 & 0.0 \\
\hline
\end{tabular}


(a) initial local time variation

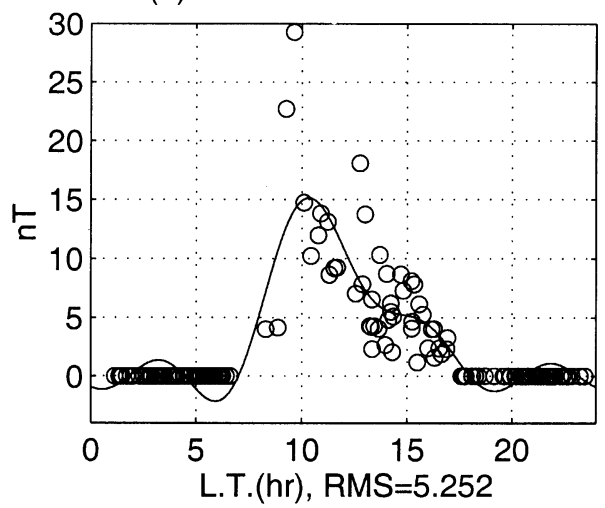

(c) after 2 normalizations

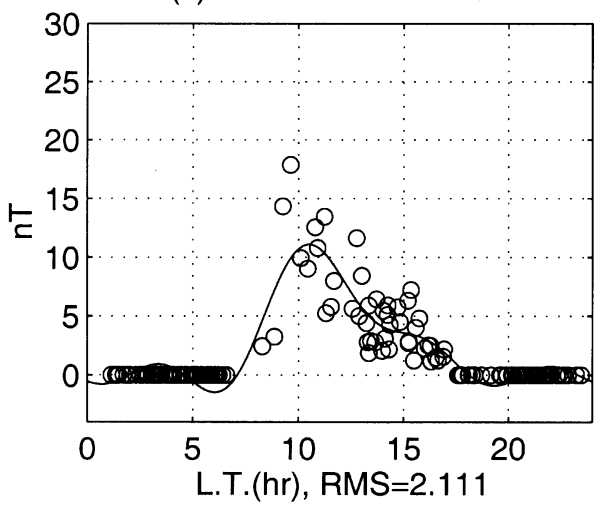

(b) after 1 normalization

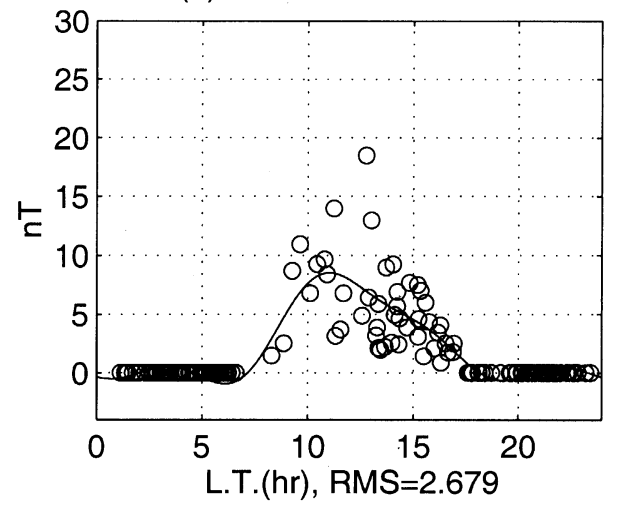

(d) comparison with previous results

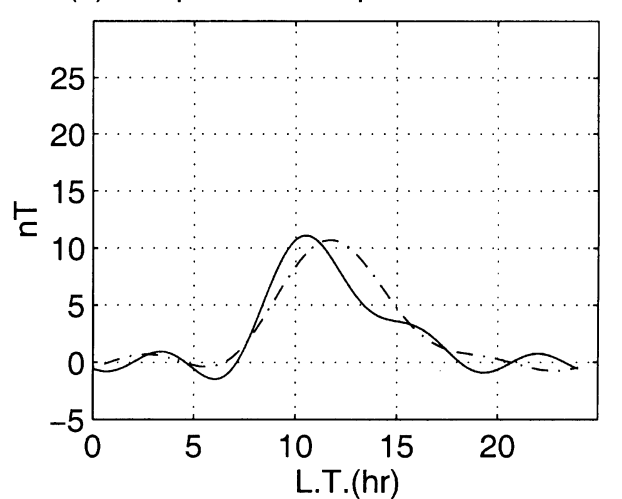

Fig. 2. The normalized curve representing the local time variation from the data set that was obtained using the direct measurement technique with the crustal correction (Direct-CC). Panel (a) shows the initial curve fit to the initial data set. Panel (b) shows that the curve after one normalization of the longitudinal variation, derived from the initial data. Panel (c) shows the curve after normalization using the longitudinal variation shown in Fig. 3 , panel (b). Panel (d) shows the comparison of the curve from panel (c) in this figure with the curve (dashed line) from Gupta and Chapman (1970) using the equation in the text. The amplitude of the Gupta and Chapman curve is scaled for comparison.

time function is represented by the line. The zero-valued data are used for the local time function because, the local time function covers the full 24-hour day. The root-mean square error (RMS) is reported below the abscissa of each graph. The RMS error did not change considerably after the first iteration of the normalization procedure. The shape of the time curve from each of the other data sets is generally consistent with the local time curve shown in Fig. 2. The local time variation curves from all four data sets include a steep rise in the amplitude of the EE in the late morning with a peak at around 1100-1130 LT and a much slower decrease through the afternoon.

In order to compare our local time results with the previous work, we note that Agu and Onwumechili (1981) found that the amplitude of the EE from the POGO satellite data is well correlated with its contemporaneous corresponding northward magnetic field value, $\Delta X$, at the dip-equatorial stations. Using this result, the normalized curve of the amplitude of the EE obtained in this study can be compared with the local time variation of the northward $(\Delta X)$ component from the observatory based models of the EE. Gupta and Chapman (1970) derived a local time variation curve using hourly geomagnetic component data from more than 100 specifically equatorial ground stations from the IGY/C (International Geophysical Year). They approximated the average observed daily variation of the northward magnetic component by the equation,

$$
\begin{aligned}
\Delta X_{\mathrm{eq}}= & 27+45 \sin \left(t+270^{\circ}\right)+24 \sin \left(2 t+90^{\circ}\right) \\
& +12 \sin \left(3 t+300^{\circ}\right)
\end{aligned}
$$

where $\Delta X_{\text {eq }}$ is the northward magnetic variation at the equator and $t$ is the local mean solar time reckoned in angle at $360^{\circ}$ per mean solar day from local mean midnight (Forbes and Lindzen, 1976). The mean value of $27 \mathrm{nT}$ is arbitrarily chosen for a baseline at midnight. The local time curve, after the second iteration of the normalization procedure described above, is compared to Eq. (5) in panel (d) of Fig. 2. The agreement here is remarkable considering that Langel (1990) estimates that the standard deviation of error estimates from all known sources in the POGO data is $5.63 \mathrm{nT}$. We do not suggest that the change in slope near 1500 local time should be taken to be significant. Further the small, negative lobes at about 0600 and 1800 local time are the result of using a low-order Fourier expansion for the local time function. Doumouya et al. (1998) used the magnetic $H$ component data from the ground equatorial stations across Africa to determine the local time variation of the EE. Our result is qualitatively consistent with their result, after scaling the amplitude of the ground data to the altitude of the satellite.

Figure 3 illustrates the results of the normalization procedure for the longitudinal variation. Figure 3 panel (a) shows 
(a) initial longitudinal variation

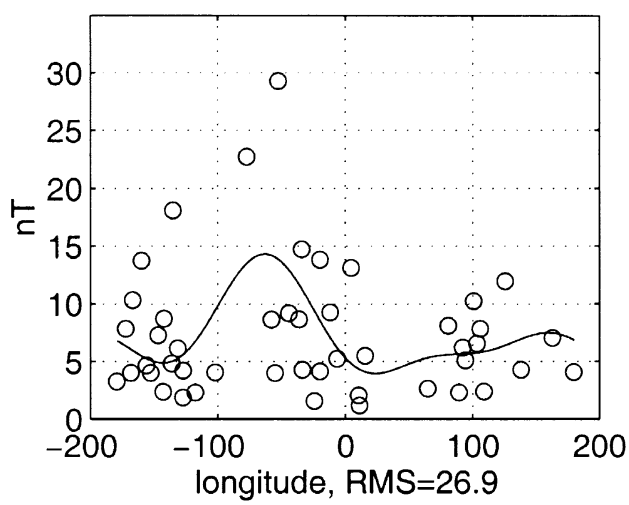

(c) after 2 normalizations

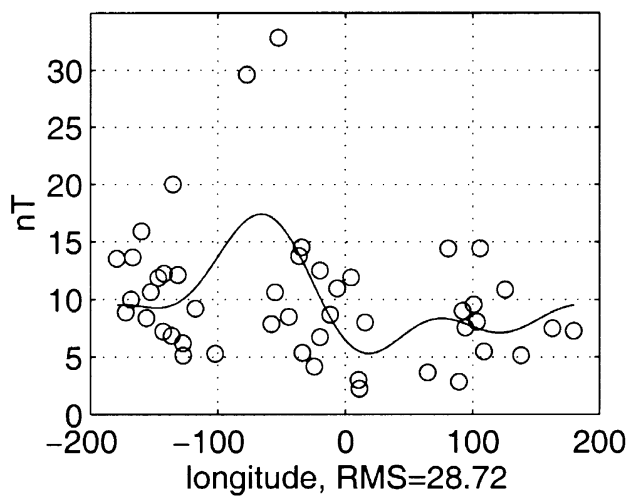

(b) after 1 normalization

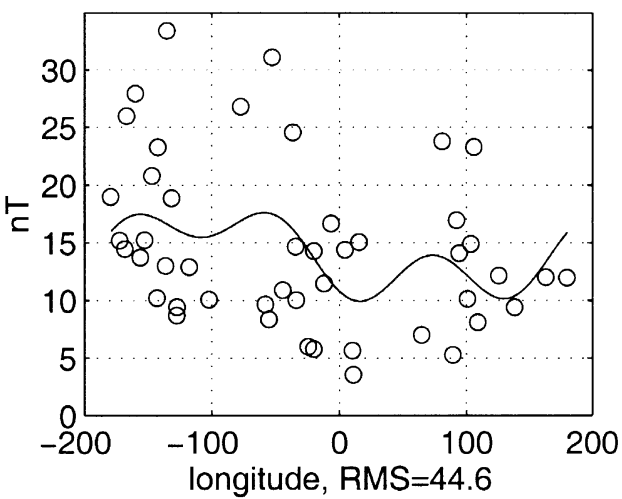

(d) comparison with previous results

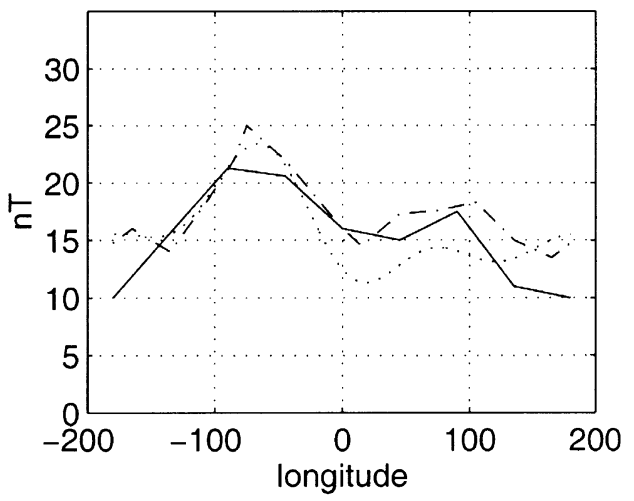

Fig. 3. The normalized curve representing the longitudinal variation from the data set in Fig. 2. Panel (a) shows the initial curve fit to the initial data set. Panel (b) shows that the curve after one normalization of the local time variation, derived from the initial data. Panel (c) shows the curve after normalization of the local time variation shown in panel (b) in Fig. 2. Panel (d) shows the comparison of the curve (dotted line) from panel (c) in this figure with the previous results. The dashed line is from Cain and Sweeney (1973) and the solid line is from Ravat and Hinze (1993). The curve in panel (c) and Ravat and Hinze's curve are normalized to Cain and Sweeney's curve at $90^{\circ} \mathrm{W}$ longitude.

the data from the direct measurement technique with the crustal correction (Direct-CC). The original data are plotted as circles and the original longitudinal function is represented by the line. The zero-valued data are not used for the longitudinal function. The normalized longitudinal variation curves, which were estimated by using a four-term harmonic expansion, are more difficult to interpret because the curves have large RMS errors, indicating that a significant fraction of the amplitude variation can not be consistently modeled with a longitudinal variation of the EE. In spite of that, it appears worthwhile to note that the pattern of the curves from the four data sets are all consistent. As a specific example, the curve after the second iteration of the normalization procedure using the Direct-CC data set (panel (c) in Fig. 3) is similar to the longitudinal variation of the EE found by Cain and Sweeney (1973) and Ravat and Hinze (1993).

Cain and Sweeney (1973) determined the longitude variation of the EE by using averaged EE magnitude normalized to a $400 \mathrm{~km}$ altitude during the interval from 0900 to 1500 local time. Ravat and Hinze (1993) also studied the longitudinal variation of the dip-latitude average of the magnetic intensities in the dip-equatorial regions by using both the Magsat dawn and dusk data sets. As shown in panel (d) of Fig. 3, these two curves are well correlated with a peak magnitude between 50 and $90^{\circ} \mathrm{W}$, and a broader, secondary maximum at $100^{\circ} \mathrm{E}$. Our longitudinal variation curve has a maximum magnitude at 50 to $80^{\circ} \mathrm{W}$, agreeing with the results of both Cain and Sweeney (1973) and Ravat and Hinze (1993). There is no secondary maximum between 100 and $110^{\circ} \mathrm{E}$ in our model. Langel et al. (1993) examined the peakto-peak amplitude of the vertical current intensity at $400 \mathrm{~km}$ altitude as a function of the longitude and also found inconclusive evidence for a secondary maximum between 100 and $110^{\circ} \mathrm{E}$. Langel et al. (1993) pointed out that Cain and Sweeney did not consider the local time variation in the data set, while the Magsat data were from dusk local time. This is a possible explanation for the difference between our result and Cain and Sweeney's. While we use POGO data, we consider local time variations. It also explains why our results are consistent with Langel et al. (1993) because, the Magsat study considered a single local time. However, within the error of the POGO data set, it is not possible for us to rule out a secondary maximum. The secondary maximum in Cain and Sweeney's model is only about $5 \mathrm{nT}$ above the average. This is beyond the resolution of the POGO data set.

We experimented with a data set that was further restricted by eliminating the satellite passes with altitudes greater than $450 \mathrm{~km}$. Applying our iterative normalization procedure to the reduced altitude data set, we obtain RMS errors are significantly reduced compared with those presented in Figs. 2 

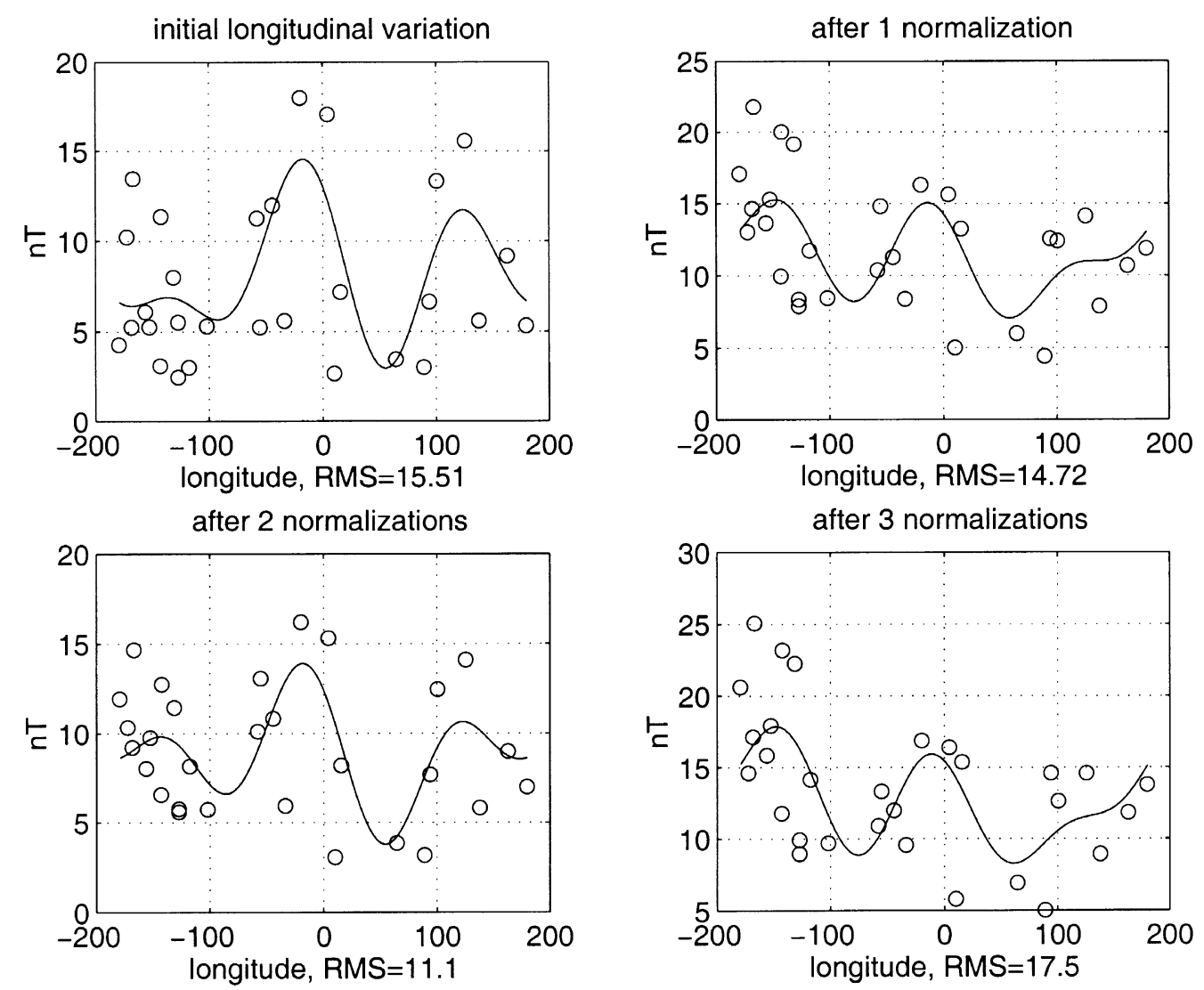

Fig. 4. The normalized curve representing the longitudinal variation from data set restricted to altitudes below $450 \mathrm{~km}$ using the same data set as in Figs. 2 and 3. The RMS error is considerably reduced compared with the full data set.

and 3. The form of the resulting local time variation curves is nearly identical to those presented in Fig. 2; however, the longitudinal variation is significantly better fit by our harmonic function. The pattern of the longitudinal variation curve differs significantly from the previous results (Fig. 4). Most notably, the primary maximum shifts from $80^{\circ} \mathrm{W}$ to $30^{\circ} \mathrm{W}$; however, we note that the region from $100^{\circ} \mathrm{W}-50^{\circ} \mathrm{W}$ has no observations to constrain the model. This experiment suggests that the amplitude of the EE over the dip equator between 450 and $800 \mathrm{~km}$ altitude may be affected by other sources. Onwumechili and Agu (1980) observed that the amplitude of the EE tends to oscillate with altitude from 400 to $800 \mathrm{~km}$, but they did not elucidate the cause of this variation. This suggests that the linear altitude correction used in this study may not be correct. It calls into question the use of the higher altitude data in EE studies. This is unfortunate because, without the higher altitude data, there are significant gaps in the longitudinal coverage of our data.

\section{Summary}

The local time variation of the amplitude of the equatorial electrojet (EE) is investigated using the POGO series satellite data. Because the POGO data are well distributed with respect to the local time, we are able to determine the local time variation of the EE amplitude from the satellite data. The resulting local time variation model compares favorably with the models presented by Gupta and Chapman (1970) and Doumouya et al., (1998). An iterative normalization procedure is developed to separate the variation of the EE with respect to the longitude from the variation with respect to the local time from the data. Even though a longitudinal variation model can not be fit to the data as well as the local time variation model, it is clear that the data contains longitudinal effects. In particular, a peak in the amplitude of the $\mathrm{EE}$ at around $90^{\circ} \mathrm{W}$ is observed. Unfortunately, the satellite tracks are not distributed uniformly in longitude. Specifically there is a lack of observations near the maximum amplitude of the EE. While the lack of, and distribution of, data limit the interpretation of the variations of the EE with respect to longitude, it appears that the variation of the amplitude of the EE with respect to longitude is on the same order as the error in the POGO data. The maximum variation of the amplitude of the EE as a function of longitude, from our analysis, is on the order of $10 \mathrm{nT}$. Considering all possible sources of error and the limited number of data points in our study, this is a difficult signal to resolve. The functions derived by fitting the data below $450 \mathrm{~km}$ altitude have significantly lower RMS errors than the functions that included all satellite passes below $800 \mathrm{~km}$ altitude. The reason for the significance of the altitude cutoff is unknown and requires further investigation.

Acknowledgments. The POGO data set was provided by R. A. Langel. Discussions with R. A. Langel are also gratefully acknowledged. The comments of two anonymous reviewers significantly improved this manuscript. 


\section{References}

Agu, C. E. and C. A. Onwumechili, Comparison of the POGO satellite and ground measurements of the magnetic field of the equatorial electrojet, J. Atmos. Terr. Phys., 43, 801-807, 1981.

Cain, J. C. and R. E. Sweeney, The POGO data, J. Atmos. Terr. Phys., 35, 1231-1247, 1973.

Doumouya, V., J. Vassal, Y. Cohen, O. Fambitakoye, and M. Menvielle, Equatorial electrojet at African longitudes: First results from magnetic measurements, Ann. Geophysicae, 16, 658-676, 1998.

Forbes, J. M., The equatorial electrojet, Rev. Geophys., 19, 469-504, 1981.

Forbes, J. M. and R. S. Lindzen, Atmospheric solar tides and their electrodynamic effects-II. The equatorial electrojet, J. Atmos. Terr. Phys., 38, 911-920, 1976.

Gupta, J. C. and S. Chapman, Manual of the coefficients of the first four harmonics of the solar and lunar daily geomagnetic variations computed from IGY/C and certain other data, NCAR Pub., MS-68-110, 1-23, 1970.

Kane, R. P., Comparison of geomagnetic changes in India and the POGO data, J. Atmos. Terr. Phys., 35, 1249-1252, 1973.

Langel, R. A., Global magnetic anomaly maps derived from POGO spacecraft data, Phys. Earth Planet. Inter., 62, 208-230, 1990.

Langel, R. A., Study of the crust and mantle using magnetic surveys by Magsat and other satellites, Proc. Indian Acad. Sci., 99, 581-618, 1990.

Langel, R. A., The use of low altitude satellite dta bases for modeling of core and crustal fields and the separation of external and internal fields, Surv. Geophys., 14, 31-87, 1993.
Langel, R. A., R. L. Coles, and M. A. Mayhew, Comparison of magnetic anomalies of lithospheric origin measured by satellite and airborne magnetometers over western Canada, Can. J. Earth Sci., 17, 876-887, 1980. Langel, R. A., M. Purucker, and M. Rajaram, The equatorial electrojet and associated currents as seen in Magsat data, J. Atmos. Terr. Phys., 55, 1233-1269, 1993.

Maeda, H., T. Iyemori, T. Araki, and T. Kamei, New evidence of a meridional current system in the equatorial ionosphere, Geophys. Res. Lett., 9, 341344, 1982.

Onwumechili, C. A., Satellite measurements of the equatorial electrojet, $J$. Geomag. Geoelectr., 37, 11-36, 1985.

Onwumechili, C. A. and C. E. Agu, General features of the magnetic field of the equatorial electrojet measured by the POGO satellites, Planet. Space Sci., 28, 1125-1130, 1980.

Rastogi, R. G., The equatorial electrojet: magnetic and ionospheric effects, in Geomagnetism, Vol. 3. Chap. 7, edited by J. A. Jacobs, pp. 461-525, Academic Press, London, 1989.

Ravat, D. and W. J. Hinze, Considerations of variations in ionospheric fields effects in mapping equatorial lithospheric Magsat magnetic anomalies, Geophys. J. Int., 113, 387-398, 1993.

Roy, M., Equatorial ionospheric currents derived from MAGSAT data, Geophys. Res. Lett., 10, 741-744, 1983.

H. R. Kim and S. D. King (e-mail: sking@purdue.edu) 
Amplitude Amplitude 